\section{Terapia endocrina neoadyuvante en cáncer de mama, una alternativa de tratamiento e investigación}

\author{
FRANCISCO ACEVEDO ${ }^{a}$, MARÍA ELISA HERRERA, \\ JORGE MADRID, CÉSAR SÁNCHEZ
}

\section{Neoadyuvant endocrine therapy in breast cancer}

Neoadjuvant chemotherapy is an accepted strategy for patients with locally advanced breast cancer. This approach increases the possibilities of conservative treatment and improves the resectability rates of initially unresectable tumors. In addition, preoperative systemic therapy allows the evaluation of prognostic and predictive factors, dynamically and in vivo. Since over $80 \%$ of these tumors express estrogen receptors $(E R)$, endocrine therapy seems a logical treatment to employ in the neoadjuvant setting. The advent of new drugs that regulate the ER function, along with the results of several clinical studies with the use of neoadjuvant endocrine therapy, support the feasibility and safety of utilizing this strategy before surgery. We herein analyze the available clinical evidence about the use of neoadjuvant therapy aiming to regulate the activity of the ER. We also discuss the value of predictive factors that could help the oncologist to select those patients most likely to benefit from this approach and the role of endocrine therapy as a research instrument.

(Rev Med Chile 2013; 141: 367-374).

Key words: Breast neoplasms; Chemotherapy, adjuvant; Receptors, estrogen.

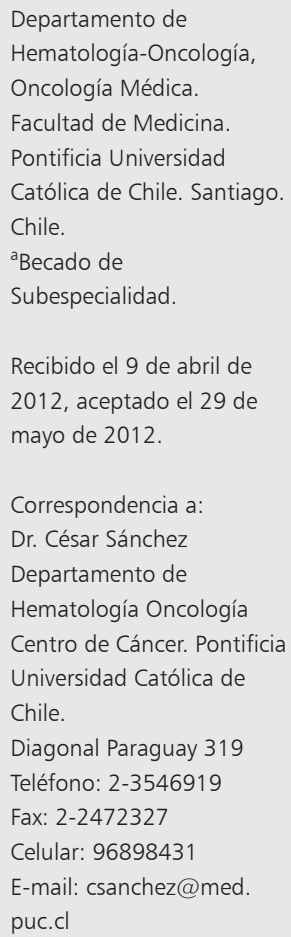

E 1 cáncer de mama (CM) es la primera causa de muerte por neoplasias en mujeres chilenas ${ }^{1}$. El amplio uso de métodos de screening, con el consiguiente aumento en las tasas de diagnóstico precoz, ha mejorado su pronóstico ${ }^{2}$. Nuevos esquemas y drogas de quimioterapia (QT) citotóxica, además del uso de anticuerpos monoclonales en tumores que expresan el receptor del factor de crecimiento epidérmico tipo 2 (HER2+), han contribuido también a esta mejoría, especialmente en tumores RE negativos y HER $2+{ }^{3}$. Sin embargo, la resistencia al tratamiento y recaídas de la enfermedad son frecuentes.

La búsqueda de mejores terapias se basa finalmente en resultados obtenidos de estudios clínicos fase III, los que comparan distintos tipos de tratamiento adyuvante (postoperatorio), teniendo como objetivo primario la sobrevida global (SG). Otra alternativa es medir los efectos de la terapia sistémica en el ámbito preoperatorio (neoadyuvancia), evaluando cambios inducidos por el tratamiento en el corto plazo sobre bio-marcadores predefinidos que están relacionados con la SG. De estos, la respuesta patológica (PR) obtenida luego de terapia neoadyuvante ha sido utilizada para predecir la SG de pacientes con $\mathrm{CM}^{4}$. Varios estudios utilizando QT preoperatoria en pacientes con tumores localmente avanzados han demostrado que el tratamiento neoadyuvante aumenta la posibilidad de conservación de la mama y mejora los resultados quirúrgicos, sin detrimento en la sobrevida, en relación al tratamiento con QT posoperatoria ${ }^{4-6}$.

Dado que más del 80\% de los CM expresan el receptor estrogénico (RE), tratamientos destinados a modular su actividad son parte fundamental en su manejo. La terapia endocrina (TE) adyuvante logra disminuir la recurrencia de la enfermedad y mejorar la SG de tumores RE+; parece lógico, por tanto, que la TE orientada molecularmente al RE y que ha demostrado beneficio en adyuvancia, se utilice también en el escenario preoperatorio en tumores $\mathrm{RE}+{ }^{7}$. 
El tamoxifeno fue inicialmente indicado en adultos mayores frágiles, con tumores localmente avanzados y contraindicaciones para el uso de tratamiento citotóxico, logrando excelente respuesta clínica y baja morbilidad ${ }^{8}$. Sin embargo, $1 / 3$ de los pacientes con tumores localizados al diagnóstico sufrirán una recurrencia de la enfermedad y hasta $50 \%$ de los pacientes con tumores avanzados RE+ serán resistentes a la TE inicial. Es necesario por tanto, descubrir nuevas formas de modulación del RE, mecanismos de resistencia y factores predictivos.

La posibilidad de PR está asociada al subtipo tumoral. Tumores RE- logran tasas de respuesta patológica completa (cPR: complete pathologic response) tras QT neoadyuvante mayores que tumores RE+, siendo esta respuesta un marcador pronóstico para tumores RE- y HER2+. Por el contrario, la frecuencia de cPR en tumores RE+ es menor al $10 \%$, por lo que en ellos son necesarios otros marcadores .

Revisaremos los resultados de estudios clínicos recientes utilizando TE neoadyuvante, su rol como herramienta de investigación y la determinación de factores predictivos.

\section{Deprivación estrogénica en mujeres posmenopáusicas}

La mayoría de los factores de riesgo clínico asociados al desarrollo de CM RE+ están asociados a niveles persistentemente altos de estrógenos (E2) $(\text { Tabla } 1)^{10}$. Debido a esto la TE tiene como objetivo bloquear la función del RE siendo hasta el momento el tratamiento más efectivo para el

\section{Tabla 1. Factores de riesgo clásicos en} cáncer de mama ${ }^{10}$

\begin{tabular}{|l|}
\hline Edad \\
\hline Antecedentes personales o familiares \\
\hline Genético \\
\hline Menarquia precoz \\
\hline Menopausia tardía \\
\hline Nuliparidad \\
\hline Terapia de reemplazo hormonal \\
\hline Consumo de alcohol \\
Obesidad \\
\hline
\end{tabular}

CM RE+, combinando eficacia, baja toxicidad y buena calidad de vida.

El tamoxifeno logra respuestas en hasta $80 \%$ de los pacientes con $\mathrm{RE} / \mathrm{RP}+{ }^{7}$. Recientemente los inhibidores de aromatasa (IA) de tercera generación han mostrado niveles de efectividad similar o superior al tamoxifeno en mujeres postmenopáusicas; sin embargo, y a pesar de mostrar una mejoría discreta en la sobrevida libre de enfermedad, los beneficios en SG no han sido consistentes, excepto en pacientes seleccionadas de alto riesgo o con linfonodos positivo ${ }^{11-13}$.

\section{TE neoadyuvante: estudios clínicos}

\section{$T E$ versus $Q T$ neoadyuvante}

Semiglazov, en un estudio aleatorio fase II estudió el efecto de la QT (doxorrubicina y paclitaxel) versus TE (anastrozol o exemestane) neoadyuvante en 239 pacientes posmenopáusicas $\mathrm{RE}$ y/o RP+ ${ }^{14}$. Luego de 3 meses de tratamiento la respuesta clínica, cPR y la sobrevida libre de progresión no fueron diferentes en ambas ramas. Tampoco hubo diferencias en resultados preliminares del GEICAM 2006-03 comparando QT vs TE neoadyuvante en pacientes postmenopáusicas ( $46 \%$ de las pacientes reclutadas), con tumores de tipo luminal definidos por inmuno-histoquímica (IHQ) (Tabla 2) ${ }^{15}$. En un análisis no planificado, tumores con Ki67 mayor al 10\% presentaron mejor respuesta clínica a la QT ( $67 \%$ vs $42 \%$; p = $0,07)$. La toxicidad grado III-IV fue significativamente mayor para el grupo que recibió QT.

Saigal y cols analizaron una cohorte de 145 pacientes postmenopáusicas con tumores $\mathrm{RE}+{ }^{19}$. La TE neoadyuvante logró niveles de cPR similares a la QT ( $5 \%$ en promedio para ambas ramas), sin diferencias en el nivel de recurrencias a 49 meses.

Así, la evidencia clínica sugiere que la TE neoadyuvante no es inferior a la QT en pacientes seleccionadas, con menor toxicidad (Tabla 3 ).

\section{IA versus tamoxifeno}

Tras la demostración de que los IA logran mayores niveles de supresión estrogénica y mayor tasa de respuestas clínicas que el tamoxifeno, se han realizado varios estudios aleatorios en mujeres jóvenes posmenopáusicas comparando estas dos drogas (Tabla 4).

Seo y cols, en un análisis combinado de estos 
Terapia endocrina neoadyuvante en cáncer de mama - F. Acevedo et al

Tabla 2. Subtipos moleculares en cáncer de mama ${ }^{16-18}$

\begin{tabular}{|c|c|c|}
\hline Subtipo molecular & Expresión IHQ & Observación \\
\hline Luminal A & $\begin{array}{l}\mathrm{ER}+, \mathrm{PR}+, \mathrm{HER} 2-, \mathrm{CK} 8 \text { y } 18 \\
\mathrm{Ki} 67<14 \%\end{array}$ & $\begin{array}{l}40 \% \text {. Tumores bien diferenciados y con buen pronóstico. } \\
\text { Poco beneficio de QT }\end{array}$ \\
\hline Luminal B & $\begin{array}{l}\mathrm{ER}+; \mathrm{PR} \pm ; \mathrm{HER} 2 \pm, \mathrm{CK} 8 \text { y } 18 \\
\mathrm{KI} 67>14 \%\end{array}$ & $\begin{array}{l}20 \% \text {. Tumores moderado o poco diferenciados. Pronóstico } \\
\text { intermedio o malo. Beneficio intermedio de QT }\end{array}$ \\
\hline HER2 + & ER-; PR-; HER2 +; Ki67 elevado & $\begin{array}{l}15 \% \text {. Tumores de alto grado. Mal pronóstico con buena } \\
\text { respuesta a QT. Susceptibles a tratamiento específico contra } \\
\text { el receptor HER2. }\end{array}$ \\
\hline Basal & ER-; PR-; HER2-; CK5/6+; EGFR+ & $\begin{array}{l}\text { 15\%. Tumores de alto grado y mal pronóstico. Son conocidos } \\
\text { como "triple negativos". Alta respuesta a QT }\end{array}$ \\
\hline Bajo en Claudina & ER-; PR-; HER2-; Ki67 intermedio & $\begin{array}{l}\text { 10\%. Diferenciación GII-III. Pronóstico intermedio, al igual } \\
\text { que su beneficio a la QT }\end{array}$ \\
\hline
\end{tabular}

Tabla 3. Estudios clínicos de neoadyuvancia. Terapia endocrina versus quimioterapia

\begin{tabular}{|c|c|c|c|c|c|}
\hline Referencia & Trata & o (n) & Duración (meses) & Respuesta clínica & PCR \\
\hline Semiglazov ${ }^{14}$ & $\begin{array}{l}\text { ANA } \\
\text { EXE } \\
\text { QT }\end{array}$ & $\begin{array}{r}(61) \\
(60) \\
(118)\end{array}$ & 3 & $\begin{array}{l}62 \% \\
67 \% \\
63 \%\end{array}$ & $\begin{array}{l}3 \% \\
6 \%\end{array}$ \\
\hline Alba $a^{15}$ & $\begin{array}{l}\text { QT } \\
\text { TE }\end{array}$ & $\begin{array}{l}(48) \\
(47)\end{array}$ & 6 & $\begin{array}{l}66 \% * \\
48 \%\end{array}$ & $\begin{array}{l}0 \% \\
3 \%\end{array}$ \\
\hline Saigal ${ }^{19, \neq}$ & $\begin{array}{l}\text { TE } \\
\text { QT }\end{array}$ & $\begin{array}{l}(46) \\
(98)\end{array}$ & 7 & & $\begin{array}{l}5 \% \\
5 \%\end{array}$ \\
\hline
\end{tabular}

ANA: anastrozol; EXE: exemestano; QT: quimioterapia; TE: terapia endocrina. N: número de pacientes. pCR: respuesta patológica completa. ${ }^{*} p=0,07$. ${ }^{\star}$ Estudio observacional.

Tabla 4. Estudios randomizados de neoadyuvancia comparando distintas terapias endocrinas

\begin{tabular}{|c|c|c|c|c|c|}
\hline Referencia & \multicolumn{2}{|c|}{ Tratamiento (n) } & Duración (meses) & Respuesta clínica & \\
\hline Eiermann ${ }^{20}$ & $\begin{array}{l}\text { LET } \\
\text { TAM }\end{array}$ & $\begin{array}{l}(154) \\
(170)\end{array}$ & 4 & $\begin{array}{l}55 \%^{\dagger} \\
36 \%\end{array}$ & $\begin{array}{l}45 \%^{\dagger} \\
35 \%\end{array}$ \\
\hline Smith ${ }^{21}$ & $\begin{array}{l}\text { ANA } \\
\text { TAM } \\
\text { Ambos }\end{array}$ & $\begin{array}{l}(113) \\
(108) \\
(109)\end{array}$ & 3 & $\begin{array}{l}37 \% \\
36 \% \\
39 \%\end{array}$ & $\begin{array}{l}44 \% \\
31 \% \\
24 \%\end{array}$ \\
\hline Cataliotti22 & $\begin{array}{l}\text { ANA } \\
\text { TAM }\end{array}$ & $\begin{array}{l}(228) \\
(223)\end{array}$ & 3 & $\begin{array}{l}50 \% \\
46 \%\end{array}$ & $\begin{array}{l}43 \%^{\dagger} \\
31 \%\end{array}$ \\
\hline Semiglazov 23 & TAM & $\begin{array}{r}E(76) \\
(75)\end{array}$ & 3 & $\begin{array}{l}76 \% \%^{+} \\
40 \%\end{array}$ & $\begin{array}{l}37 \%^{\dagger} \\
20 \%\end{array}$ \\
\hline Ellis ${ }^{24}$ & $\begin{array}{l}\text { EXE } \\
\text { LET } \\
\text { ANA }\end{array}$ & & 4 & $\begin{array}{l}61 \% \\
71 \% \\
67 \%\end{array}$ & $65 \% \%^{\ddagger}$ \\
\hline Masuda25 & $\begin{array}{l}\text { ANA } \\
\text { TAM }\end{array}$ & & 6 & $\begin{array}{l}70 \%^{\dagger} \\
50 \%\end{array}$ & \\
\hline
\end{tabular}

LET: letrozol. ANA: anastrozol. EXE: exemestano. TAM: tamoxifeno. ${ }^{\dagger}$ Diferencia estadísticamente significativa. 讘 relación al grupo total. Si se consideran sólo pacientes inoperables este valor se eleva a 75\%. 
trabajos (1.160 pacientes), confirma que los IA obtiene mayores tasas de respuesta clínica (RR, 1,29; 95\% CI, 1,14-1,47; p < 0,001), ecográfica (RR, 1,29; 95\% CI, 1,10-1,51; P = 0,002) y de cirugía conservadora $(\mathrm{RR}, 1,36$; 95\% $\mathrm{CI}, 1,16-1,59$; $\mathrm{p}<0,001)^{26}$.

Otros estudios fase II han confirmado estos hallazgos con respuestas clínicas de 37 a 100\%, sin embargo, los niveles de cPR con TE rara vez superan el $5 \%{ }^{27-30}$.

\section{Marcadores de respuesta a terapia endocrina neoadyuvante: factores predictivos}

Clásicamente la elección de tratamiento sistémico en pacientes con CM no metastásico se ha basado en la evaluación de perfiles de riesgo basales, combinando factores tales como la edad, co-morbilidades, TN (tamaño tumoral (T) y compromiso nodal axilar $(\mathrm{N})$ ), además de parámetros histopatológicos: presencia de RE y/o receptor de progesterona (RP), HER2, invasión vascular y/o linfática, y grado de diferenciación del tumor. Sin embargo, pacientes con la misma etapa según la clasificación TNM y un perfil de IHQ similar no responden uniformemente al tratamiento y cursan con variadas evoluciones a largo plazo ${ }^{18}$.

Es necesario, por tanto el descubrimiento de nuevos bio-marcadores que permitan personalizar el tratamiento del CM. Estos bio-marcadores (perfiles moleculares o IHQ, vías de señalización, proteínas, imágenes, etc.) pueden ser categorizados como pronósticos (Tabla 5) o predictivos (Tabla 6) ${ }^{18}$.

Analizaremos marcadores predictivos clásicos de TE, aquellos que han sido obtenidos del análisis de perfiles genéticos y del modelo de TE neoadyuvante.

\section{Bio-marcadores clásicos en CM}

Receptor estrogénico. La presencia del RE es el factor clínico-patológico más importante para predecir la respuesta a TE. El EBCTCG (Early Breast Cancer Trialists' Collaborative Group) ha actualizado los datos de un meta-análisis que incluye 21.457 mujeres con CM precoz, provenientes de estudios aleatorios comparando el uso de tamoxifeno adyuvante por 5 años versus placebo ${ }^{8}$. Este estudio demuestra que el tamoxifeno reduce el riesgo de mortalidad en $30 \%$, manteniendo su beneficio más allá de los 10 años desde el diagnóstico, (RR 0,70; p < 0,00001). Pacientes con tumores RE negativos no se beneficiaron del uso de TE, pero tumores con valores de RE tan bajos como $10 \mathrm{fmol} / \mathrm{mg}$ de proteína aun mostraron mejor sobrevida con el uso de TE.

Receptor de progesterona. Dado que la expresión del RP depende del RE, en pacientes con $\mathrm{RE}-\mathrm{y} \mathrm{RP}+$ positivo, se sugiere repetir el examen $\mathrm{y}$ tratar del mismo modo que tumores con RE+. En el meta-análisis citado previamente el beneficio de la TE adyuvante fue similar en pacientes con RE+ versus aquellos con RE- y $\mathrm{RP}+{ }^{8}$. Tumores $\mathrm{RE}+/$ $\mathrm{RP}$ - pueden reflejar la activación de vías de señales de crecimiento y los IA podrían ser más efectivos que el tamoxifeno en esta población ${ }^{31}$.

HER 2 neu. La presencia de HER2 es un marcador de resistencia endocrina. Pacientes tratados con IA, con tumores RE+HER2+, tienen peor evolución que aquellos RE+HER2-32.

KI67. La expresión de Ki67 refleja la proporción de células en proliferación. En el IMPACT, un valor elevado de Ki67 fue asociado a una peor sobrevida en tumores tratados con IA, aunque esta diferencia no fue estadísticamente significativa $^{21}$. En el BIG 1-98, el Ki67 elevado fue el único factor que predijo el beneficio de los IA por sobre el tamoxifeno ${ }^{12}$. En el mismo estudio un score

\section{Tabla 5. Factores pronósticos en cáncer de mama $^{18}$}

Tamaño tumoral (T)

Compromiso ganglionar (N)

Grado histológico

Receptor de estrógeno y progesterona

HER2

Marcadores de proliferación celular (ej: Ki67)

Perfiles genéticos, ej: Oncotype DX® y MammaPrint ${ }^{\circledR}$

Tabla 6. Factores predictivos en cáncer de mama $^{18}$

Receptor estrógeno

Receptor progesterona

HER2 
compuesto que utilizó el estatus del RE, RP, HER2, grado nuclear (GN) y Ki67 fue capaz de predecir el beneficio de IA o tamoxifeno como terapia adyuvante en pacientes con CM RE+. Un análisis posterior del estudio P024, el letrozol demostró ser significativamente más efectivo que el tamoxifeno en reducir la proliferación tumoral ${ }^{33}$.

\section{Valor predictivo de perfiles genéticos en CM}

Perou describió cuatro clases moleculares de CM, llamadas subtipos intrínsecos (Tabla 2), que se corresponden con la clasificación clínicopatológica clásica ${ }^{16}$. Utilizando diferentes métodos para el estudio y clasificación de perfiles genéticos se han definido varios perfiles de riesgo. Aunque originalmente diseñados para evaluar el pronóstico del CM, podrían también predecir la respuesta a TE.

Algunos de estos perfiles genéticos (Tabla 5) han sido validados en estudios clínicos y están disponibles comercialmente ${ }^{34-36}$. Aquellos que requieren tejido fijado en parafina son factibles de usar en la práctica clínica habitual. Uno de los más utilizados es el Oncotype DX que calcula un score de recurrencia basado en la expresión de 16 genes relacionados a $\mathrm{CM} \mathrm{RE}+$. En un análisis retrospectivo del NSABP-14, este score logró predecir la sobrevida libre de recurrencia a 10 años $^{36}$.

Pacientes con score de riesgo alto cursan con un riesgo de recurrencia de 30\% cuando reciben sólo tamoxifeno. Mientras este hallazgo podría indicar resistencia a la terapia, también puede estar determinado por el mal pronóstico basal, independiente del tratamiento. Si bien este test es ampliamente utilizado en la toma de decisiones, especialmente en pacientes $\mathrm{RE}+$, sin compromiso nodal, existe evidencia de que variables clínicas y patológicas más clásicas proveen información pronóstica independiente del score ${ }^{37-38}$.

El TAILORx está analizando prospectivamente el valor del Oncotype DX en la decisión de tratamiento sistémico en tumores $\mathrm{RE}+{ }^{39}$.

\section{TE neoadyuvante como modelo para investigar factores predictivos}

Mientras los bio-marcadores descritos anteriormente ayudan a determinar sensibilidad a TE, no identifican a tumores con resistencia primaria o adquirida. Los perfiles de expresión génica disponibles en clínica identifican subgrupos pronósticos, pero no están diseñados para evaluar factores predictivos. La clasificación propuesta por Perou no abarca la heterogeneidad del CM dentro de los mismos subtipos y, al ser una evaluación basal del tumor, no da cuenta de cambios inducidos por el tratamiento ${ }^{16}$.

El modelo de estudio basado en la TE neoadyuvante da cuenta de la sensibilidad in vivo y permite el estudio de bio-marcadores dinámicos (miden la diferencia entre el valor inicial y el observado intra-tratamiento), tal como se evalúa y ajusta la terapia anti-hipertensiva o diabética según los controles de presión arterial y glicemia durante la terapia.

Los estudios de neoadyuvancia parecen ser una solución a la gran cantidad de pacientes, tiempo y costos asociados al desarrollo de estudios clínicos fase III en adyuvancia; en ellos, un marcador substituto medido precozmente, ayudaría a determinar factores predictivos y pronósticos. Interesantemente valores dinámicos resultan de mejor correlación con el pronóstico global que mediciones basales. El NSABP B-27 buscó la relación entre niveles de pCR y mejorías en la SG y libre de enfermedad en el escenario de QT neoadyuvante ${ }^{4}$. Este estudio confirmó que la pCR es un factor predictivo de SG. Pero, mientras los niveles de pCR luego de QT preoperatoria pueden superar el $50 \%$ en pacientes con tumores triple negativos o HER2+, estos niveles distan mucho de los obtenidos en tumores RE $+{ }^{40}$. Por tanto, otros bio-marcadores han debido ser investigados en tumores RE+.

El índice de proliferación celular se ha establecido como un buen marcador del efecto de la TE neoadyuvante. Este se ha evaluado a través de varias técnicas, incluyendo el índice mitótico, la fracción de fase $\mathrm{S}$, la incorporación de bromodeoxiuridina y la tinción por IHQ del antígeno nuclear Ki67. La mayoría de los centros utiliza esta última técnica por la facilidad en su medición y utilidad demostrada en varios estudios prospectivos $^{41}$.

En el IMPACT una reducción en los niveles de Ki67 luego de 2 a 12 semanas de TE neoadyuvante con anastrozol, predijo la sobrevida libre de enfermedad observada en el ATAC (Arimidex, Tamoxifen, Alone or in Combination). Mientras el IMPACT analizó datos de pacientes reclutados 
y seguidos por meses, el ATAC requirió miles de pacientes, seguidos por años ${ }^{1121}$.

Ellis definió un score pronóstico post-tratamiento utilizando distintos factores obtenidos a través del análisis multivariado del estudio P024 y luego validados en el estudio IMPACT ${ }^{42}$. Tumores que lograron disminuir el Ki67, reducir su tamaño original (respuesta patológica), con ganglios negativos posterior al tratamiento $y$ que mantuvieron la positividad del RE (versus aquellos que la perdieron) presentaron un riesgo de recurrencia menor al 3\%, por lo que pudieran no beneficiarse de QT. Este algoritmo es un buen ejemplo de las ventajas de la TE neoadyuvante y de cómo la presión selectiva inducida por el tratamiento puede seleccionar, precozmente, pacientes con resistencia al tratamiento; sin embargo, aun es necesario mayor seguimiento para determinar su utilidad a largo plazo, debido al patrón de recaídas tardías de los tumores luminales.

\section{Conclusiones}

Estudios recientes en mujeres posmenopáusicas muestran que la TE neoadyuvante logra respuestas similares o superiores a la QT en tumores $\mathrm{RE}+$, sin la toxicidad derivada de esta última.

La TE neoadyuvante posibilita la evaluación dinámica de los efectos inducidos por la deprivación estrogénica y permite individualizar y ajustar el tratamiento basado en la evaluación clínica, patológica, guiada por imágenes o molecular durante la terapia.

La TE preoperatoria parece una atractiva y eficaz forma de manejo para nuestro medio. Permite iniciar el tratamiento sistémico precoz de tumores RE+ durante el lapso previo a la cirugía y además es una herramienta de bajo costo para el estudio de factores predictivos y pronósticos en tumores RE+.

\section{Referencias}

1. Departamento de Estadística e Información en Salud 2009. Ministerio de Salud. Gobierno de Chile. Disponible en: www.deis.cl. [Consultado el 25 de marzo de 2012].

2. Berry DA, Cronin KA, Plecritis SK, Fryback DG, Clarke $\mathrm{L}$, Zelen $\mathrm{M}$, et al. Effect of screening and adjuvant therapy on mortality from breast cancer. N Engl J Med 2005;
353 (17): 1784-92.

3. Yin W, Jiang Y, Shen Z, Shao Z, Lu J. Trastuzumab in the adjuvant treatment of HER2-positive early breast cancer patients: a meta-analysis of published randomized controlled trials. PLoS One 2011; 6 (6): e21030.

4. Bear HD, Anderson S, Smith RE, Geyer CE Jr, Mamounas EP, Fisher B, et al. Sequential preoperative or postoperative docetaxel added to preoperative doxorubicin plus cyclophosphamide for operable breast cancer: National Surgical Adjuvant Breast and Bowel Project Protocol B-27. J Clin Oncol 2006; 24 (13): 2019-27.

5. Wolmark N, Wang J, Mamounas E, Bryant J, Fisher B. Preoperative chemotherapy in patients with operable breast cancer: nine-year results from National Surgical Adjuvant Breast and Bowel Project B-18. J Natl Cancer Inst Monogr 2001; 2001 (30): 96-102.

6. Van der Hage JA, van de Velde CJ, Julien JP, TubianaHulin M, Vandervelden C, Duchateau L. Preoperative chemotherapy in primary operable breast cancer: results from the European Organization for Research and Treatment of Cancer trial 10902. J Clin Oncol. 2001; 19 (22): 4224-37.

7. Peto R, Davies C, Godwin J, Gray R, Pan HC, Clarke M, et al. Comparisons between different polychemotherapy regimens for early breast cancer: meta-analyses of longterm outcome among 100,000 women in 123 randomised trials. Early Breast Cancer Trialists' Collaborative Group (EBCTCG). Lancet 2012; 379 (9814): 432-44.

8. Gazet JC, Markopoulos C, Ford HT, Coombes RC, Bland JM, Dixon RC. Prospective randomised trial of tamoxifen versus surgery in elderly patients with breast cancer. Lancet 1988; 1 (8587): 679-81.

9. Darb-Esfahani S, Loibl S, Müller BM, Roller M, Denkert C, Komor M, et al. Identification of biology-based breast cancer types with distinct predictive and prognostic features: role of steroid hormone and HER2 receptor expression in patients treated with neoadjuvant anthracycline/taxane-based chemotherapy. Breast Cancer Res. 2009; 11 (5): R69.

10. Benson JR, Jatoi I, Keisch M, Esteva FJ, Makris A, Jordan C. Early Breast Cancer. Lancet 2009; 373 (9673): 146379.

11. Cuzick J, Sestak I, Baum M, Buzdar A, Howell A, Dowsett $M$, et al. Effect of anastrozole and tamoxifen as adjuvant treatment for early-stage breast cancer: 10-year analysis of the ATAC trial. Lancet Oncol 2010; 11 (12): 1135-41.

12. Regan M, Neven P, Giobbie-Hurder A, Goldhirsch A, Ejlertsen B, Mauriac L, et al. Assessment of letrozole and tamoxifen alone and in sequence for postmenopausal women with steroid hormone receptor-positive breast 
cancer: the BIG 1-98 randomised clinical trial at $8 \cdot 1$ years median follow-up. Lancet Oncol 2011; 12 (12): 1101-8.

13. Goss PE, Ingle JN, Martino S, Robert NJ, Muss HB, Piccart MJ, et al. Randomized trial of letrozole following tamoxifen as extended adjuvant therapy in receptorpositive breast cancer: updated findings from NCIC CTG MA.17. J Natl Cancer Inst 2005; 97 (17): 1262-71.

14. Semiglazov VF, Semiglazov VV, Dashyan GA, Ziltsova EK, Ivanov VG, Bozhok AA, et al. Phase 2 randomized trial of primary endocrine therapy versus chemotherapy in postmenopausal patients with estrogen receptorpositive breast cancer. Cancer 2007; 110 (2): 244-54.

15. Alba E, Calvo L, Albanell J, De la Haba J, Chacon J, Arcusa A, et al. Chemotherapy (CT) versus hormone therapy (HT) as neoadjuvant treatment in luminal breast cancer: A multicenter, randomized phase II study (GEICAM/2006-03). J Clin Oncol 2010; 28: 15s (abstr $500)$.

16. Perou CM, Sorlie T, Eisen MB, van de Rijn M, Jeffrey SS, Rees CA, et al. Molecular portraits of human breast cancer. Nature 2000; 406 (6797): 747-52.

17. Prat A, Parker JS, Karginova O, Fan C, Livasy C, Herschkowitz JI, et al. Phenotypic and molecular characterization of the claudin-low intrinsic subtype of breast cancer. Breast Cancer Res 2010; 12 (5): R68.

18. Reis-Filho JS, Pusztai L. Gene expression profiling in breast cancer: classification, prognostication and prediction. Lancet 2011; 378 (9805): 1812-23.

19. Saigal K, Saeed AM, Hurley J, Takita C, Reis IM, Zhao $\mathrm{W}$, et al. Pathologic response and locoregional outcome in postmenopausal ER positive breast cancer patients receiving neoadjuvant endocrine therapy vs cytotoxic chemotherapy. ASTRO 2011; abstract 181.

20. Eiermann W, Paepke S, Appfelstaedt J, Llombart-Cussac A, Eremin J, Vinholes J, et al. Preoperative treatment of postmenopausal breast cancer patients with letrozole: A randomized double-blind multicenter study. Ann Oncol 2001; 12 (11): 1527-32.

21. Smith IE, Dowsett M, Ebbs SR, Dixon JM, Skene A, Blohmer JU, et al. Neoadjuvant treatment of postmenopausal breast cancer with anastrozole, tamoxifen, or both in combination: the Immediate Preoperative Anastrozole, Tamoxifen, or Combined with Tamoxifen (IMPACT) multicenter double-blind randomized trial. J Clin Oncol 2005; 23 (22): 5108-16.

22. Cataliotti L, Buzdar AU, Noguchi S, Bines J, Takatsuka $\mathrm{Y}$, Petrakova $\mathrm{K}$ et al. Comparison of anastrozole versus tamoxifen as preoperative therapy in postmenopausal women with hormone receptor-positive breast cancer: the Pre-Operative "Arimidex" Compared to Tamoxifen
(PROACT) trial. Cancer 2006; 106 (10): 2095-103.

23. Semiglazov V, Kletsel A, Semiglazov V, Zhiltzova E, Ivanov V, Dashyan G, et al. Exemestane (E) vs tamoxifen ( $\mathrm{T}$ ) as neoadjuvant endocrine therapy for postmenopausal women with ER+ breast cancer (T2N1-2, T3N0-1, T4N0M0). J Clin Oncol 2005; 23: 16 S (abstr. 530).

24. Ellis MJ, Buzdar A, Unzeitig GW, Esserman L, Leitch M, Deshryver K, et al. ACOSOG Z1031: A randomized phase II trial comparing exemestane, letrozole, and anastrozole in postmenopausal women with clinical stage II/ III estrogen receptor-positive breast cancer. J Clin Oncol 2010; 28: 18s (abstr. LBA513).

25. Masuda N, Sagara Y, Kinoshita T, Iwata H, Nakamura S, Yanagita $Y$, et al. Neoadjuvant anastrozole versus tamoxifen in patients receiving goserelin for premenopausal breast cancer (STAGE): a double-blind, randomized phase 3 trial. Lancet Oncol 2012; 13 (4): 345-52.

26. Seo JH, Kim YH, Kim JS. Meta-analysis of pre-operative aromatase inhibitor versus tamoxifen in postmenospausal woman with hormone receptor-positive breast cancer. Cancer Chemother Pharmacol 2009; 63 (2): 261-6.

27. Barnadas A, Gil M, González S, Tusquets I, Muñoz M, Arcusa A, et al. Exemestane as primary treatment of oestrogen receptor-positive breast cancer in postmenopausal women: a phase II trial. Br J Cancer 2009; 100 (3): 442-9.

28. Krainick U, Astner A, Jonat W, Wallwiener D. Phase II study to define safety and efficacy of exemestane as preoperative therapy for postmenopausal patients with primary breast cancer final results of the German Neoadjuvant Aromasin Initiative (GENARI). Breast Cancer Res Treat. 2003; 83 (suppl 1): S55. Abstract 239.

29. Dixon JM, Renshaw L, Bellamy C, Stuart M, HoctinBoes G, Miller WR. The effects of neoadjuvant anastrozole (Arimidex) on tumor volumen in postmenospausal women with breast cancer: a randomized, double-blind, single-center study. Clin Cancer Res 2000; 6 (6): 222935.

30. Milla-Santos A, Milla L, Calvo N, Portella J, Rallo L, Casanovas JM, et al. Anastrozole as neoadjuvant therapy for patients with hormone-dependent, locally-advanced breast cancer. Anticancer Res 2004; 24 (2C): 1315-8.

31. Viale G, Regan MM, Dell'Orto P, Mastropasqua G, Maiorano E, Rasmussen BB, et al. Which patients benefit most from adjuvant aromatase inhibitors? Results using a composite measure of prognostic risk in the BIG 1-98 randomized trial. Ann Oncol 2011; 22 (10): 2201-7.

32. Dowsett M, Allred C, Knox J, Quinn E, Salter J, Wale C, et al. Relationship between quantitative estrogen and progesterone receptor expression and human epidermal 
growth factor receptor 2 (HER-2) status with recurrence in the Arimidex, Tamoxifen, Alone or in Combination trial. J Clin Oncol 2008; 26 (7): 1059-65.

33. Ellis $\mathrm{M}, \mathrm{Ma} \mathrm{C}$. Letrozole in the neoadjuvant setting: the P024. Breast Cancer Res Treat. 2007; 105 suppl 1: 33-43.

34. Van't Veer LJ, Dai H, van de Vijver MJ, He YD, Hart AA, Mao M, et al. Gene expression profiling predicts clinical outcome of breast cancer. Nature 2002; 415 (6871): 530-6.

35. Wang Y, Klijn JG, Zhang Y, Sieuwerts AM, Look MP, Yang F, et al. Gene-expression profiles to predict distant metastasis of lymph-node-negative primary breast cancer. Lancet 2005; 365 (9460): 671-79.

36. Paik S, Shak S, Tang G, Kim C, Baker J, Cronin M, et al. A multigene assay to predict recurrence of tamoxifentreated, node-negative breast cancer. $\mathrm{N}$ Engl J Med 2004; 351 (27): 2817-26.

37. Tang G, Shak S, Paik S, Anderson SJ, Constantino JP, Geyer CE, et al. Comparison of the prognostic and predictive utilities of the 21-gene Recurrence Score and Adjuvant! for women with node-negative, ER-positive breast cancer: results from NSABP B-14 and NSABP B-20. Breast Cancer Res Treat 2011; 127 (1): 133-42.

38. Cuzick J, Dowsett M, Pineda S, Wale C, Salter J, Quinn $\mathrm{E}$, et al. Prognostic value of a combined estrogen receptor, progesterone receptor, Ki-67, and human epider- mal growth factor receptor 2 immunohistochemical score and comparison with the genomic health recurrence score in early breast cancer. J Clin Oncol 2011; 29 (32): 4273-8.

39. Phase III randomized study of adjuvant combination chemotherapy and hormonal therapy versus adjuvant hormonal therapy alone in women with previously resected axillary node-negative breast cancer with various levels of risk for recurrence (TAILORx Trial). Disponible en: http://www.cancer.gov/clinicaltrials/ ECOG-PACCT-1. (Consultado el 25 de marzo de 2012).

40. Guarneri V, Broglio K, Kau SW, Cristofanilli M, Buzdar AU, Valero V, et al. Prognostic value of pathologic complete response after primary chemotherapy in relation to hormone receptor status and other factors. J Clin Oncol 2006; 24 (7): 1037-44.

41. de Azambuja E, Cardoso F, de Castro G, Colozza M, Mano MS, Durbecq V, et al. Ki-67 as prognostic marker in early breast cancer: a meta-analysis of published studies involving 12,155 patients. Br J Cancer 2007; 96 (10): 1504-13.

42. Ellis MJ, Tao Y, Luo J, A'Hern R, Evans DB, Bhatnagar AS, et al. Outcome prediction for estrogen receptor-positive breast cancer based on postneoadjuvant endocrine therapy tumor characteristics. J Natl Cancer Inst 2008; 100 (19): 1380-8. 